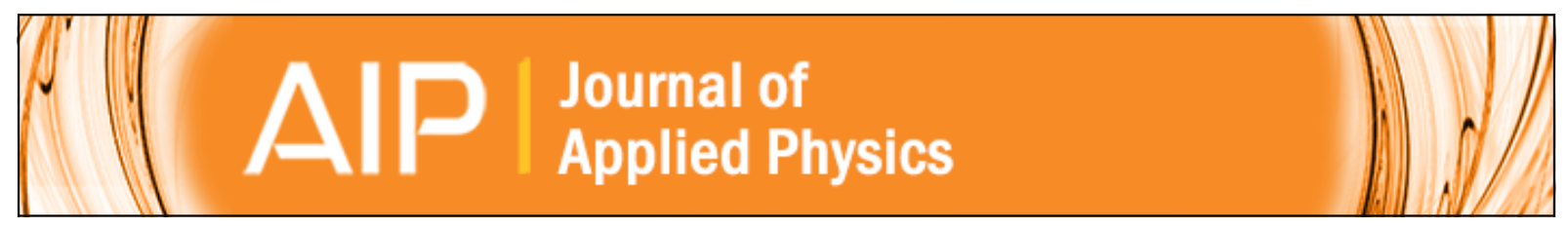

\title{
Hyperbolic heat conduction with temperature-dependent thermal conductivity
}

D. E. Glass, M. N. Özișik, D. S. McRae, and Brian Vick

Citation: Journal of Applied Physics 59, 1861 (1986); doi: 10.1063/1.336413

View online: http://dx.doi.org/10.1063/1.336413

View Table of Contents: http://scitation.aip.org/content/aip/journal/jap/59/6?ver=pdfcov

Published by the AIP Publishing

\section{Articles you may be interested in}

Temperature-Dependent Electrical Conductance of Bi Nanowires

Chin. J. Chem. Phys. 28, 79 (2015); 10.1063/1674-0068/28/cjcp1409164

Predictive calculation of the lattice thermal conductivity with temperature-dependent vibrational parameters J. Appl. Phys. 112, 123506 (2012); 10.1063/1.4769429

Temperature-dependent thermal conductivity of nanorod-based nanofluids

Appl. Phys. Lett. 89, 083111 (2006); 10.1063/1.2338424

The function estimation in measuring temperature-dependent thermal conductivity in composite material J. Appl. Phys. 78, 6949 (1995); 10.1063/1.360462

Heat Flow in Connected Media of Different Temperature-Dependent Conductivities

Rev. Sci. Instrum. 34, 1218 (1963); 10.1063/1.1718182

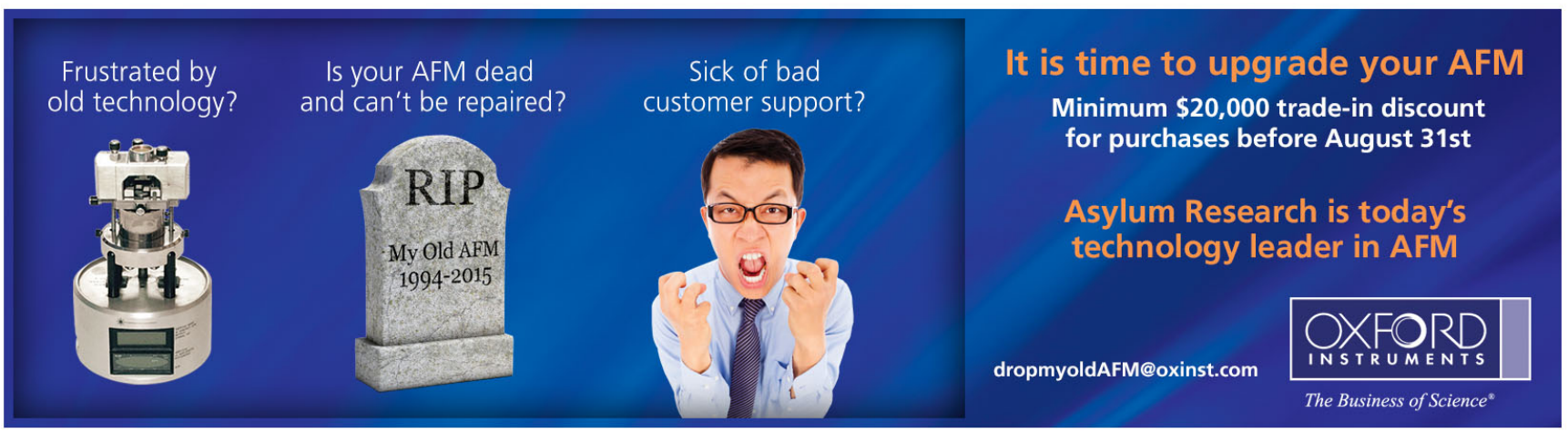




\title{
Hyperbolic heat conduction with temperature-dependenthemal conductiviny
}

\author{
D. E. Giass, M. N. Özişix, and D. S. McRae \\ Mechanical and Aerospace Engineering Department, North Carolina State University, Raleigh, North \\ Carolina 27695-7910 \\ Brian Vick \\ Mechanical Engineering Department, Virginia Polytechnic Institute and State University, Blacksburg. \\ Vinginia 24061
}

(Received 19 August 1985; accepted for publication 1 November 1985)

\begin{abstract}
Hyperbolic heat conduction in a semi-infinite slab with temperature-dependent thermal conductivity is studied numerically, and the results are compared with those obtained from the classical parabolic equation for the following cases: (a) constant applied temperature at $x=0.0$, (b) constant applied heat flux at $x=0.0$, and (c) a pulsed heat source released instanteously at $t=0.0$ in the region $0.0<x<\Delta x$ adjacent to an insulated boundary. In addition to changing the temperature profiles, the nonlinear thermal conductivity also altered the speed of the thermal front. An increase in the thermal conductivity increased the wave speed, while a decrease in the thermal conductivity decreased the wave speed.
\end{abstract}

\section{INTRODUCTION}

According to the hyperbolic heat-conduction model, ${ }^{1-7}$ thermal waves travel with a finite wave speed, in contrast to the nonphysical infinite speed of propagation predicted by the classical parabolic heat-conduction equation. The existence of finite wave speeds has been demonstrated experimentally, ${ }^{8,9}$ and has been studied theoretically with analytic and numerical approaches. ${ }^{10-17}$ Despite its shortcoming, the classical parabolic heat-conduction equation successfully predicts the temperature and heat-xux distribution for most practical purposes, except for extremely short times and for temperatures near absolute zero. The effects of temperaturedependent thermal conductivity on the temperature distribution in solids have been studied with the parabolic model, but no work appears to be available for the case of the hyperbolic model. In this work, MacCormack's predictor-corrector scheme is used to solve the hyperbolic heat-conduction equation numerically in order to study the effects of temperature-dependent thermal conductivity.

\section{FORMULATHON}

The hyperbolic heat-conduction equation results from the use of the non-Fourier heat-flux equation, first proposed by Vernotte ${ }^{1}$ in the form

$$
\tau \frac{\partial q(x, t)}{\partial t}+q(x, t)=-k \frac{\partial T(x, t)}{\partial x},
$$

where $q(x, t)$ is the heat flux, $T(x, t)$ the temperature, and $x$ and $t$ are the space and time variables. In addition, the relaxation time $\tau$ defined as $\tau=\alpha / c^{2}$ is assumed constant where $\alpha$ is the thermal diffusivity and $c$ is the speed of propagation. The thermal conductivity $k$ is assumed to vary with temperature linearly as

$$
k=k_{0}\left(1+\beta^{\prime} T\right),
$$

where $\beta^{\prime}$ is the temperature coefficient and $k_{0}$ is the reference thermal conductivity. Clearly, when the relaxation time $\tau$ is zero, the non-Fourier heat-flux Eq. (1) reduces to the classical Fourier heat-fiux equation as

$$
q(x, t)=-k \frac{\partial T(x, t)}{\partial x} .
$$

Although the significant terms in the heat-flux equation are different for the hyperbolic and parabolic problems, the energy equation remains unchanged, and is given by

$$
-\frac{\partial q(x, t)}{\partial x}+g(x, t)=\rho C_{p} \frac{\partial T(x, t)}{\partial t},
$$

where $\rho$ is the density, $C_{p}$ is the constant pressure specific heat, and $g(x, t)$ is a volumetric energy source.

Attention is now focused on a semi-infinite slab, $0<x<\infty$, initially at a uniform temperature of $T_{i}$. In order to observe the influence of a temperature-dependent thermal conductivity, three different types of thermal disturbances are considered:

(1) A constant temperature $T_{0}$ is applied at the surface $x=0.0$ of a region with no energy generation;

(2) a constant heat flux $f_{0}$ is applied at the surface $x=0.0$ of a region with no energy generation;

(3) the boundary surface at $x=0.0$ is kept insulated, and a pulsed energy source releases its energy spontaneously at $t=0.0$. Such an energy source is represented mathematically in the form

$$
g(x, t)=\left\{\begin{array}{ll}
\left(g_{0} / \Delta x\right) \delta(t) & 0<x<\Delta x \\
0 & \Delta x<x
\end{array},\right.
$$

where $g_{0}$ is a reference energy generation rate per unit volume, and $\delta(t)$ is the Dirac delta function.

Equations (4) and (1) are given, respectively, in the dimensionless form as

$$
\begin{aligned}
& \frac{\partial \theta}{\partial \xi}+\frac{\partial Q}{\partial \eta}-\frac{1}{2} S=0, \\
& \frac{\partial Q}{\partial \xi}+\frac{\partial}{\partial \eta}[\theta(1+0.5 \beta \theta)]+2 Q=0,
\end{aligned}
$$


where $\theta, Q$, and $S$ are, respectively, the dimensionless temperature, heat flux, and energy generation rate per unit volume and their definitions vary according to the type of problem considered as shown in Table I. In addition, $\eta=\frac{1}{2} c_{0} x / \alpha_{0}$ and $\xi=\frac{1}{c_{0}} c_{0}^{2} t / \alpha_{0}$ are the dimensionless space and time variables with $\alpha_{0}$ and $c_{0}$ being the reference thermal diffusivity and speed of propagation, and $\beta=\beta^{\prime}\left(k_{0} c_{0} / f_{0} \alpha_{0}\right)$ is the dimensionless temperature coefficient. Equations (6) are now written in vector form as

$$
\frac{\partial \mathbf{E}}{\partial \xi}+\frac{\partial \mathbf{F}}{\partial \eta}+\mathbf{H}=0
$$

where

$$
\begin{aligned}
& \mathbb{E}=\left[\begin{array}{l}
\theta \\
Q
\end{array}\right], \\
& \mathbb{F}=\left[\begin{array}{c}
Q \\
\theta(1+0.5 \beta \theta)
\end{array}\right], \\
& \mathbb{H}=\left[\begin{array}{c}
-\frac{t}{2} S \\
2 Q
\end{array}\right] .
\end{aligned}
$$

The dimensionless form of the parabolic heat-conduction equation with thermal conductivity varying linearly with temperature is given by

$$
\frac{\partial \theta}{\partial \xi}=\frac{1}{2} \frac{\partial}{\partial \eta}\left[(1+\beta \theta) \frac{\partial \theta}{\partial \eta}\right]+\frac{1}{2} S
$$

\section{NUMERICAL ANALYSIS}

The hyperbolic problems considered here all have discontinuities at the thermal wave front. MacCormack's method, which is a second-order accurate explicit scheme, has been shown to handle these moving discontinuities quite well, ${ }^{16,17}$ and is thus used in the present investigation. When MacCormack's method is applied to Eq. (7), the following finite difference formulation results ${ }^{18}$ :

Predictor:

$$
\tilde{\mathbf{E}}_{i}^{n+1}=E_{i}^{n}-\frac{\Delta \xi}{\Delta \eta}\left[\mathbb{F}_{i+1}^{n}-\mathbb{F}_{i}^{n}\right]-\Delta \xi \mathbb{H}_{i}^{n},
$$

\section{Corrector:}

$$
\begin{aligned}
\mathbb{E}_{i}^{n+1}= & \frac{1}{2}\left[\mathbb{E}_{i}^{n}+\tilde{\mathbf{E}}_{i}^{n+1}-\frac{\Delta \xi}{\Delta \eta}\left(\tilde{F}_{i}^{n+1}-\tilde{\mathbb{F}}_{i-1}^{n+1}\right)\right. \\
& \left.-\Delta \xi \tilde{\mathbb{H}}_{i}^{n+1}\right] .
\end{aligned}
$$

Here, the subscript $i$ denotes the grid point in the space domain, superscript $n$ denotes the time level, the tilde denotes the predicted value at the time level $n+1$, and $\Delta \eta$ and $\Delta \xi$ are the space and time steps, respectively. In this formulation, forward differencing is used in the predictor, while backward differencing is used in the corrector.

The stability bound for this method, when applied to a linear equation, is $v<1.0$, where $v=\Delta \xi / \Delta \eta$ is the Courant number. However, due to the nonlinear nature of the present equations, the stability bound is reduced. As the Courant number becomes smaller, the effect of odd derivative truncation-error terms becomes larger, and oscillations occur in the vicinity of discontinuities in the solution. Also, since even derivative terms are found in the truncation error, gradients of wave fronts may be reduced. These solution errors can be reduced by including the leading truncation-error terms of the modified equation in the finite difference scheme such that the leading error terms are effectively canceled. ${ }^{19}$ In the present analysis, the modified equation used is similar to the one for constant thermal conductivity, ${ }^{17} \mathrm{ex}$ cept for the definition of the $F$ vector [Eq. (7c) ], and is given by

$$
\frac{\partial \mathbb{E}}{\partial \xi}+\frac{\partial \mathbf{F}_{0}}{\partial \eta}+\mathbf{H}=\mathbf{0}
$$

\begin{tabular}{|c|c|c|c|c|c|}
\hline $\begin{array}{l}\text { Case } \\
\text { No. }\end{array}$ & $\begin{array}{l}\text { Boundary } \\
\text { condition at } \\
\eta=0^{\circ}\end{array}$ & $\begin{array}{l}\text { Initial } \\
\text { condition for } \\
\xi=0\end{array}$ & $\begin{array}{l}\text { Source } \\
\text { term }\end{array}$ & $\begin{array}{l}\text { Definition of } \\
\theta(\eta, \xi)\end{array}$ & $\begin{array}{l}\text { Definition of } \\
Q(\eta, \xi)\end{array}$ \\
\hline \multirow[t]{2}{*}{1.} & $\theta=1$ & $\theta=0$ & $S=0$ & $\theta=\frac{T(x, t)-T_{i}}{T_{0}}$ & $Q=\frac{q}{T_{0} k_{0} \mathcal{C}_{0} / \alpha_{0}}$ \\
\hline & $\left(\frac{\partial Q}{\partial \eta}=0\right)$ & $Q=0$ & & & \\
\hline \multirow[t]{2}{*}{2.} & $Q=1$ & $\theta=0$ & $S=0$ & $\theta=\frac{T(x, t)-T_{I}}{a_{d} f_{0} / k_{0} c_{0}}$ & $Q=\frac{q}{f_{0}}$ \\
\hline & $\left(\frac{\partial \theta}{\partial \xi}=-\frac{\partial Q}{\partial \eta}\right)$ & $Q=0$ & & & \\
\hline \multirow[t]{2}{*}{3.} & $Q=0$ & $\theta=0$ & $\begin{array}{l}S=1 . \delta(\xi) \\
\text { in } 0<\eta<\Delta \eta\end{array}$ & $\theta=\frac{T(x, t)-T_{1}}{g_{0} c_{0} / k_{0}}$ & $Q=\frac{g}{g_{0} \mathcal{C}_{0}^{2} / \alpha_{0}}$ \\
\hline & $\left(\frac{\partial}{\partial \eta}[\theta(1+0.5 \beta \theta)]=0\right)$ & $Q=0$ & & & \\
\hline
\end{tabular}

where

$$
\begin{aligned}
& \mathbb{F}_{0}=\mathbb{F}-\Delta_{2}-\Delta_{3}, \\
& \Delta_{2}=\frac{(\Delta \eta)^{2}}{3 !}\left[\mathbb{F}_{n n}-v^{2} F^{\prime}\left(F^{\prime} F_{n n}\right)\right], \\
& \Delta_{3}=\frac{(\Delta \eta)^{3}}{4 !}\left\{3 v F^{\prime}\left[\left(I-v^{2} F^{\prime} F^{\prime}\right) \mathbb{F}_{n n n}\right]\right\}
\end{aligned}
$$

TABLE I. Boundary and initial conditions and the definitions of $\theta(\eta, \xi)$ and $Q(\eta, \xi)$ for the problems investigated.

- The additional conditions shown in parentheses are derived from Eqs. (6a) and (6b) by utilizing the actual boundary conditions and are needed for numerical computations. 
$I$ is the identity matrix and the Jacobian matrix $F^{\prime}$ is defined as

$$
F^{\prime}=\frac{\partial \mathbf{F}}{\partial \mathbf{E}}=\left[\begin{array}{ll}
\frac{\partial F_{1}}{\partial E_{1}} & \frac{\partial F_{1}}{\partial E_{2}} \\
\frac{\partial F_{2}}{\partial E_{1}} & \frac{\partial F_{2}}{\partial E_{2}}
\end{array}\right] .
$$

The hyperbolic problem defined by the above modified equation is integrated by MacCormack's method.

The parabolic problem, specified by Eq. (8), is solved by the Crank-Nicolson method, which results when $\lambda=0.5$ in the general implicit/explicit scheme given by

$$
\begin{aligned}
\theta_{i}^{n+1}= & \theta_{i}^{n}+\lambda \frac{\Delta \xi}{2(\Delta \eta)^{2}}\left[\left(1+\beta \theta_{i+1 / 2}^{n}\right)\left(\theta_{i+1}^{n+1}-\theta_{i}^{n+1}\right)\right. \\
& \left.-\left(1+\beta \theta_{i-1 / 2}^{n}\right)\left(\theta_{i}^{n+1}-\theta_{i-1}^{n+1}\right)\right] \\
& +(1-\lambda) \frac{\Delta \xi}{2(\Delta \eta)^{2}} \\
& \times\left[\left(1+\beta \theta_{i+1 / 2}^{n}\right)\left(\theta_{i+1}^{n}-\theta_{i}^{n}\right)\right. \\
& \left.-\left(1+\beta \theta_{i-1 / 2}^{n}\right)\left(\theta_{i}^{n}-\theta_{i-1}^{n}\right)\right]
\end{aligned}
$$

where

$\theta_{i+1 / 2}=\frac{1}{2}\left(\theta_{i+1}+\theta_{i}\right)$,

$\theta_{i-1 / 2}=\frac{1}{2}\left(\theta_{i}+\theta_{i-1}\right)$.

\section{RESULTS AND DISCUSSION}

The hyperbolic and parabolic heat-conduction problems are solved numerically by using the system of equations given by Egs. (10) and (11), respectively, for each of the three different cases described previously. For constant thermal conductivity, a Courant number as high as $v=0.98$ can be used in solving the hyperbolic heat conduction equation. However, for each of the numerical solutions presented here, a Courant number of $v=0.714$ was used to allow direct comparison of solutions for all cases. This value of the Courant number was determined for the $\beta=0.25$ solution, since it required a lower Courant number than that for the $\beta=-0.25$ solution. This is due to the fact that for $\beta=-0.25$, the nonlinear term acts as a dissipative term, and does not significantly reduce stability, while for $\beta=0.25$, the sign of the nonlinear term is changed, and a destabilizing effect is observed. Each of the numerical solutions was obtained using 1000 time intervals from $\xi=0.0$ to the final time, $\xi=1.0$. The linear problems (i.e., $\beta=0.0$ ) are calculated analytically (Case 1, Ref. 15; Case 2, Ref. 11; Case 3, Ref. 12).

The nonlinear, parabolic solutions for $\beta= \pm 0.25$ were calculated using the Crank-Nicolson method. For cases involving constant temperature and constant heat flux at the boundary, a spatial interval of $\Delta \eta=0.02$ was used, along with a second-order accurate representation for the boundary conditions. In the case of the pulse problem, the spatial interval was taken equal to the pulse width. The results of the numerical and analytic solutions are presented in Figs. 1-3.

Figure 1 shows the effect of temperature-dependent thermal conductivity on the temperature distribution for both the hyperbolic and parabolic problems for the case of a

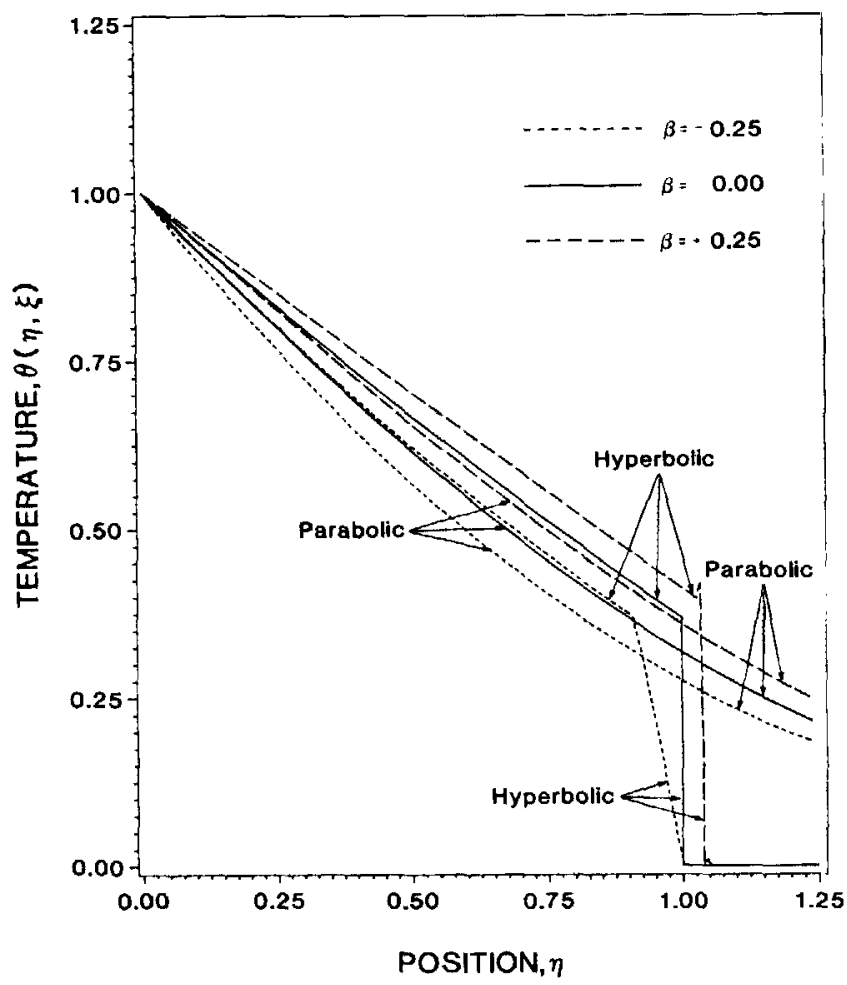

FIG. 1. Hyperbolic and parabolic temperature distributions at time $\xi=1.0$ in a semi-infinite medium with temperature-dependent thermal conductivity and an applied surface temperature at $\eta=0.0$.

constant surface temperature $\theta_{0}$ equal to unity. For all values of $\beta$, the temperature predicted by the parabolic solution is less than that predicted by the hyperbolic solution. The wave speed is affected by a nonzero value of $\beta$, such that it is decreased with $\beta=-0.25$ and increased with $\beta=0.25$. This is to be expected since by definition the wave speed depends on thermal conductivity $k(T)$, hence on $T$ as

$$
c=\sqrt{\frac{\alpha}{\tau}}=\sqrt{\frac{k_{0}\left(1+\beta^{\prime} T\right)}{\rho C_{p} \tau}}=c_{0} \sqrt{1+\beta^{\prime} T}
$$

since $\tau$ is assumed to be constant. The variation of the wave speed with temperature as shown by Eq. (12) affects the temperature gradients at the wave front. Analogous gradients occur in compressible fluid flow wave motion. In the case of $\beta<0.0$, the wave speed decreases with increased temperature, thereby causing wave speed and temperature gradient to reduce through the wave front. The leading portion of the $\beta=-0.25$ front coincides with the location of the $\beta=0.0$ wave, since local wave speed for the leading portion is not significantly different from that of the $\beta=0.0$ wave. To verify that the location of the trailing portion of the $\beta=-0.25$ wave was correct, the location was obtained from a separate integration using wave speed calculated from the observed temperature. The results of this integration agreed well with the location of the $\beta=-0.25$ wave as shown in Fig. 1. For $\beta>0.0$, wave speed increases as temperature increases. Therefore, the high-temperature portion of the wave determines wave speed for the entire front and the large gradient character of the wave front is maintained. 
Figure 2 shows the temperature profiles for the case of a constant heat flux of strength unity applied at the surface $\eta=0.0$ of a semi-infinite medium with temperature-dependent thermal conductivity. Again, the temperatures obtained from the parabolic solution are less than those from the corresponding hyperbolic solution. In this figure, the curves for different values of $\beta$ intersect each other in both the hyperbolic and parabolic solutions, and the intersection occurs at approximately the same location. The reason for the crossing of the temperature profiles is that a lower value of thermal conductivity (i.e., $\beta=-0.25$ ) results in both a larger temperature gradient and a higher surface temperature, while a higher thermal conductivity results in a smaller temperature gradient and a lower surface temperature. As in Fig. 1, the gradient of the wave front in the hyperbolic solution for $\beta=-0.25$ is reduced due to the wave speed reduction through the front. The wave speeds for the $\beta= \pm 0.25$ solutions are affected by the nonlinear thermal conductivity in the same manner that was pointed out in reference to Fig. 1.

Figure 3 shows the effect of different values of $\beta$ on the temperature distribution at time $\xi=1.0$ in a semi-infinite medium. The temperature distribution results from a pulsed energy source at time $\xi=0.0$ giving rise to an initial temperature of unity over the region $0 \leqslant \eta \leqslant \Delta \eta$ for $\Delta \eta=0.0098$. At time $\xi=1.0$, the pulse has advanced to the position $\eta \simeq 1.0$ in the hyperbolic solutions, in a manner similar to that of the thermal fronts in Figs. 1 and 2. Note that the parabolic solutions significantly underestimate the temperature in the medium at the location of the pulse and that the effects of temperature dependence of thermal conductivity are not distinguishable in graphical form. This is due to the fact that the very low temperatures have an almost negligible effect on the thermal conductivity as defined by Eq. (2). In

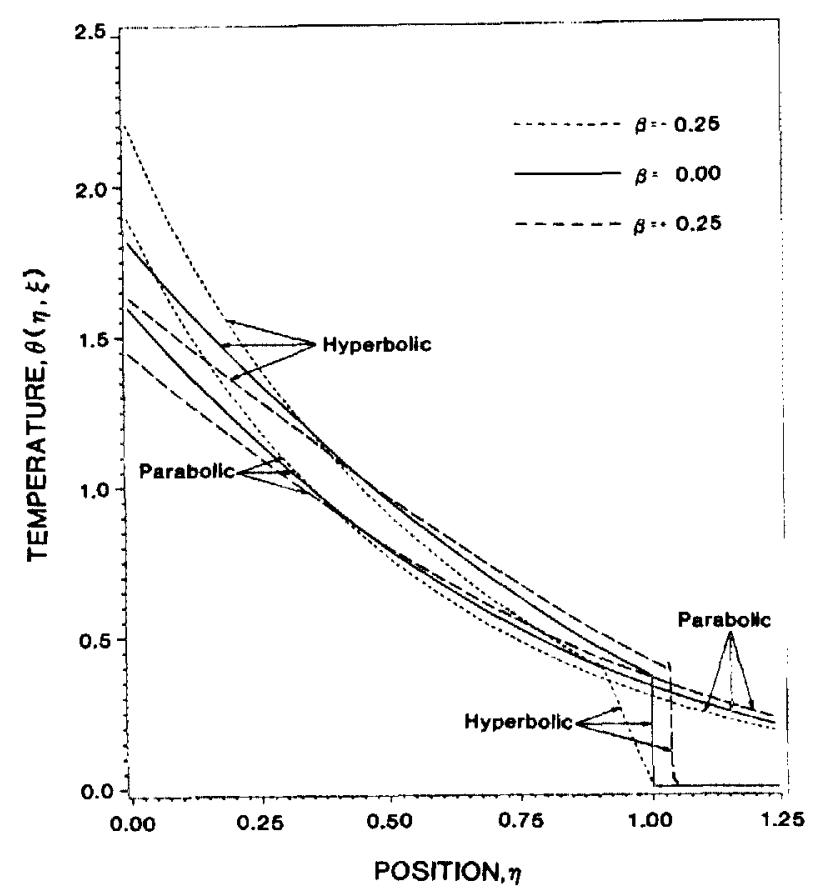

FIG. 2. Hyperbolic and parabolic temperature distributions at time $\xi=1.0$ in a semi-infinite medium with temperature-dependent thermal conductivity and an applied surface heat flux at $\eta=0.0$.

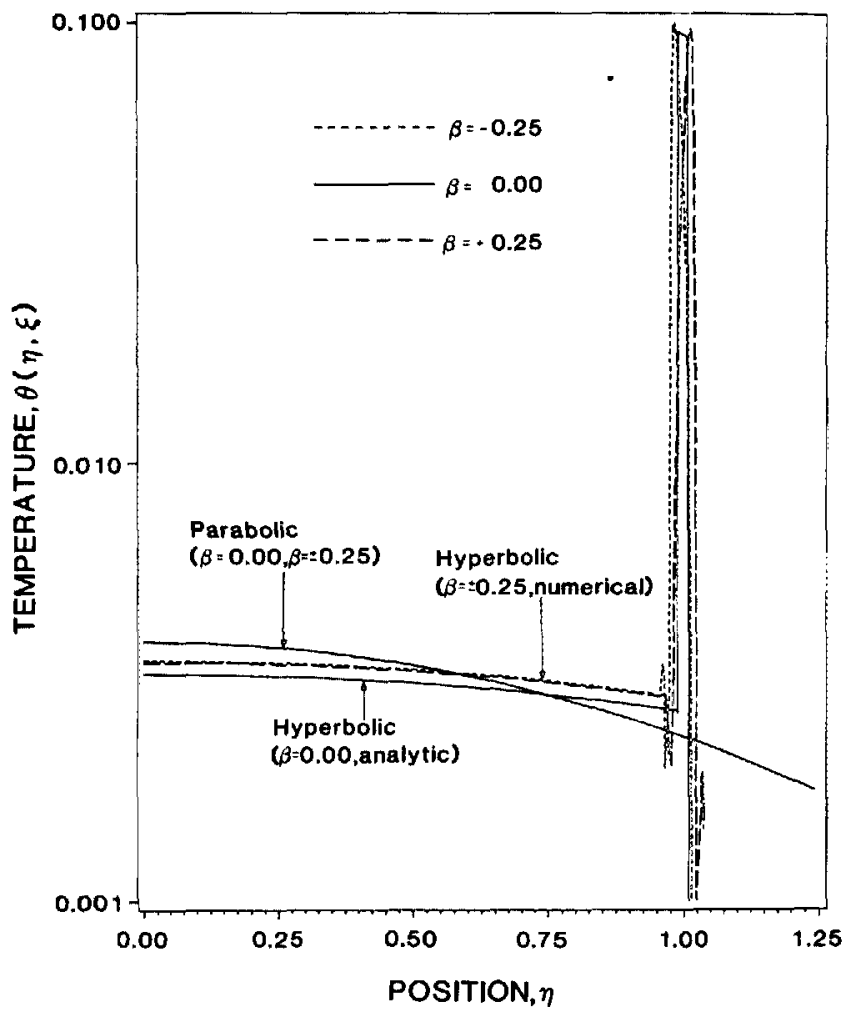

FIG. 3. Hyperbolic and parabolic temperature distributions at time $\xi=1.0$ in a semi-infinite medium with temperature-dependent thermal conductivity and a pulse heat source applied in the region $0<\eta<\Delta \eta$ at time $\xi=0.0$.

the solution of the hyperbolic problem, a slight temperature difference is observed in the region behind the pulse for $\beta= \pm 0.25$. The difference in the hyperbolic residual temperatures for the cases $\beta=0.0$ and $\beta= \pm 0.25$ is due to the fact that the solution for $\beta=0.0$ is calculated analytically, whereas for $\beta= \pm 0.25$, it is calculated numerically. It should also be pointed out that the log scale used in the figure tends to magnify the temperature differences that are present. Such a difference between the numerical and analytic temperatures in the wake of the pulse has been observed in a previous work. ${ }^{16}$ As expected, numerical oscillations are observed on each side of the pulse. The speed of the thermal front is affected by the dependence of the thermal conductivity on temperature in a similar manner as in Figs. 1 and 2. However, the wave speed is not altered as much as in Figs. $l$ and 2 because the lower temperatures have a much smaller effect on the temperature-dependent thermal conductivity, which in turn affects the wave speed.

In conclusion, it has been observed that the temperature-dependent thermal conductivity affects the temperature distributions of both the hyperbolic and parabolic solutions, but also affects the speed of the thermal front in the hyperbolic solution. The wave speed increases as the thermal conductivity increases, and decreases as thermal conductivity decreases. This change is consistent with what is predicted by the definition of the wave speed.

\section{ACKHOWEEDGASENT}

This work was supported through the National Science Foundation Grant No. MEA 8313301. 
1P. Vernotte, C. R. Acad. Sci. 246, 3154 (1958)

${ }^{2}$ M. Chester, Phys. Rev. 131, 2013 (1963).

${ }^{3}$ H. D. Weymann, Am. J. Phys. 35, 488 (1967).

${ }^{4}$ M. E. Gurtin and A. C. Pipkin, Arch. Ration. Mech. Anal. 31, 113 (1968).

${ }^{5}$ M. J. Maurer, J. Appl. Phys. 40, 5123 (1969).

${ }^{\circ}$ Y. Taitel, Int. J. Heat Mass Transfer 15, 369 (1972).

${ }^{7}$ A. V. Luikov, V. A. Bubnov, and I. A. Soloview, Int. J. Heat Mass Transfer 19, 245 (1976).

${ }^{8}$ V. Peshkov, J. Phys. VIII, 381 (1944).

${ }^{9}$ B. Bertman and D. J. Sandiford, Sci. Am. 222, 92 (1970).

${ }^{10}$ K. J. Baumeister and T. D. Hamill, J. Heat Transfer 191, 543 (1969).
${ }^{11}$ M. J. Maurer and H. A. Thompson, J. Heat Transfer 95, 284 (1973).

${ }^{12}$ B. Vick and M. N. Özişik, J. Heat Transfer 105, 902 (1983).

${ }^{13}$ M. N. Özişik and B. Vick, Int. J. Heat and Mass Transfer 27, 1845 (1984).

${ }^{14}$ D. C. Wiggert, J. Heat Transfer 99, 35 (1977).

${ }^{15}$ G. F. Carey and M. Tsai, Numerical Heat Transfer 5, 309 (1982).

${ }^{16}$ D. E. Glass, M. N. Öziģik, D. S. McRae, and B. Vick, Numerical Heat Transfer 8, 497 (1985).

${ }^{17}$ D. E. Glass, M. N. Özişik, and B. Vick, Int. J. Heat Mass Transfer 28, 1823 (1985).

${ }^{18} \mathrm{D}$. A. Anderson, J. C. Tannehill, and R. H. Pletcher, Computational Fluid Mechanics and Heat Transfer (Hemisphere, New York, 1984), Chap. 4.

${ }^{19}$ G. H. Klopfer and D. S. McRae, AIAA J. 21, 487 (1983). 